\title{
Costos por la demora quirúrgica en la fractura de cadera por fragilidad
}

\author{
Costs of surgical delay in fragility hip fracture
}

\begin{abstract}
Juan Carlos Viveros-García, * Beatriz Rodríguez-Sánchez, ${ }^{\ddagger}$ Lizbeth Saraí Baldenebro-Lugo, $\$$ Evelyn Alina Guillermo-Nuncio," Hugo Rolando Nieto-Sandoval," Enrique Vázquez-Cantero** *Medicina Interna y Ortogeriatría. Hospital Regional ISSSTE León, México. ORCID: https://orcid.org/0000-0001-9965-7253: ₹Doctora en Economía de la Salud. Facultad de Comunicación y Humanidades, Universidad Camilo Cela, Madrid, España; $\$$ Geriatra. Jefatura de Investigación Clínica. Hospital Regional ISSSTE León, México; "Residente de cuarto año de Traumatología y Ortopedia. Hospital Regional ISSSTE, León, México;" Traumatología y Ortopedia. Profesor Titular de la Especialidad de Traumatología y Ortopedia. Hospital Regional ISSSTE, León, México; ${ }^{\star *}$ Coordinación de Calidad. Hospital Regional ISSSTE León, México.
\end{abstract}

\begin{abstract}
Resumen
Introducción: Las fracturas de cadera tienen una alta tasa de complicaciones, mortalidad y riesgo de generar dependencia; además los costos son muy elevados. Existen factores modificables, como la demora quirúrgica, que incrementan de manera innecesaria los costos en salud. Disminuir la demora quirúrgica puede ahorrar recursos y mejorar resultados asistenciales. Objetivos: Analizar los costos generados por la demora quirúrgica en fractura de cadera y compararlos con los que tuvieron cirugía temprana. Material y métodos: Estudio observacional, retrospectivo y transversal en el Hospital Regional ISSSTE León entre 2017 y 2018. Se realizó un análisis de costos directos atribuidos a la fractura de cadera en los pacientes con cirugía en menos de 48 horas y se comparó contra cirugía en más de 48 horas. Resultados: El costo total de la fractura de cadera fue de $\$ 124,956$ pesos mexicanos, con una diferencia estadísticamente significativa de $\$ 25,539.10$ entre el grupo con cirugía en $<48$ vs $>48$ horas. Conclusión: La demora quirúrgica en fractura de cadera genera un costo innecesario al instituto, además de tener efectos negativos en la salud de los pacientes. Se deben generar procesos para operar a los pacientes en las primeras 48 horas.
\end{abstract}

Palabras clave: Fractura de cadera, costos, demora prequirúrgica.

\begin{abstract}
Introduction: Hip fracture has a high rate of complications, mortality and increases the risk of developing functional decline. In addition to this, the costs are very high. There are modifiable factors such as surgical delay that unnecessarily raise health costs. Decreasing surgical delay can reduce costs and improve healthcare outcomes. Objectives: To analyze the costs generated by the surgical delay in hip fracture and compare them with early surgery. Material and methods: Observational, retrospective, cross sectional study at Hospital Regional ISSSTE León between 2017 to 2018. An analysis of direct costs attributed to the hip bill was carried out in patients with surgery in less than 48 hours and it was compared against surgery in more than 48 hours. Results: The total cost of the hip fracture was $\$ 124,956$ Mexican pesos, with a statistically significant difference of $\$ 25,539.10$ between the group with surgical delay vs early surgery. Conclusion: The surgical delay in hip fracture generates an unnecessary cost to the institute in addition to having negative effects on the health of the patients. Policies must be generated to operate on patients in the first 48 hours.
\end{abstract}

Keywords: Hip fracture, costs, presurgical delay.

Correspondencia:

Juan Carlos Viveros-García

E-mail: drviveros.geriatria@gmail.com

Recibido: 02-07-2021. Aceptado: 06-07-2021.
Citar como: Viveros-García JC, Rodríguez-Sánchez B, BaldenebroLugo LS, Guillermo-Nuncio EA, Nieto-Sandoval HR, VázquezCantero E. Costos por la demora quirúrgica en la fractura de cadera por fragilidad. Orthotips. 2021; 17 (4): 195-201. https://dx.doi. org/10.35366/102216 


\section{Introducción}

En las últimas décadas ha habido un envejecimiento acelerado. ${ }^{1}$ En México el último censo poblacional reportó 10.4 millones de adultos mayores, y se espera que para 2050 esta cifra aumente a 36.4 millones, además también se ha incrementado la esperanza vida, que se proyecta alcance los 82 años. ${ }^{2}$ Como consecuencia de este fenómeno demográfico se espera que las fracturas por fragilidad se multipliquen $^{3}$ convirtiéndose en un problema de salud pública, de las cuales la más grave es la fractura de cadera (FC) ${ }^{4}$

La FC se considera una entidad catastrófica debido a su asociación a un decremento en la calidad de vida, generación de dependencia, alta morbimortalidad y costos. ${ }^{5-7}$ Esta entidad se ha incrementado sustancialmente en las últimas décadas, particularmente en los países en vías de desarrollo. ${ }^{8}$ La International Osteoporosis Foundation (IOF) ha estimado que existen alrededor de 1.6 millones de FC al año. ${ }^{9}$ Esta tendencia se incrementará en los siguientes años, esperando que se tripliquen los casos para $2050 .{ }^{8}$

Uno de los puntos que influyen de manera significativa en el pronóstico de la fractura de cadera es el tiempo entre el ingreso al servicio de urgencias y la cirugía. ${ }^{10,11}$ En México se ha reportado una demora quirúrgica de 140 horas en hospitales públicos contra 40 horas en privados. ${ }^{12}$ Hay tendencia a coincidir en que la demora quirúrgica es uno de los factores que afectan de forma significativa los resultados clínicos y la supervivencia, ${ }^{11-13}$ además de incrementar de manera considerable los costos. ${ }^{14}$ En este rubro el estándar de calidad es tener un tiempo de espera entre 36 y 48 horas. ${ }^{15-17}$ En México hay pocas publicaciones cuyo tema central sea la demora quirúrgica en FC. Los trabajos que la reportan encontraron una media de entre 102 y 140 horas, las principales causas son administrativas o de logística, y no atribuidas a la condición clínica del paciente. ${ }^{12,18}$

En cuanto a los costos, algunos trabajos han reportado que la atención a la FC en nuestro país cuesta entre $\$ 5,803$ y $\$ 37,636$ dólares estadounidenses. ${ }^{19-21}$ En 2009 los sistemas de salud gastaron aproximadamente $\$ 118,033,675$ dólares estadounidenses, y proyectaban un incremento cercano a los 4,000 millones en los siguientes años. ${ }^{22}$ Por otra parte, el Instituto Nacional de Rehabilitación estimó en 2008 un costo de atención a la FC de $\$ 1,729.85$ dólares, incluyendo costos quirúrgicos, prótesis, nueve días de hospitalización y auxiliares en el diagnóstico. ${ }^{21}$
Hasta donde los autores tienen conocimiento no existen trabajos sobre el costo atribuido a la demora quirúrgica en México.

El objetivo primario del estudio fue analizar el costo asociado a la demora quirúrgica en los pacientes con fractura de cadera por fragilidad. Los objetivos secundarios fueron conocer el costo general atribuido a la atención directa en FC en el Hospital Regional ISSSTE León, además de conocer el posible ahorro por paciente con demora quirúrgica si se operara en un lapso menor de 48 horas así como los resultados asistenciales. Por último, conocer las principales causas de demora quirúrgica en nuestro hospital.

\section{Material y métodos}

Estudio retrospectivo, transversal, analítico y comparativo realizado en el Hospital Regional ISSSTE León, que es un hospital de concentración para los trabajadores del gobierno. Recibe pacientes de cuatro estados de la República mexicana con un promedio anual de 55 fracturas de cadera por año. Cuenta con programas de residencias médicas de traumatología y ortopedia, medicina interna y geriatría. En 2017 se atendía a los pacientes con un modelo tradicional sin intervenciones de geriatría. En 2018 se inició con un modelo interconsultante de ortogeriatría con intervención de un internista y geriatra en el turno matutino de lunes a viernes. El resto de los cuidados asistenciales recaían en el servicio de traumatología y ortopedia.

Se incluyeron todos los pacientes mayores de 60 años ingresados al servicio de traumatología y ortopedia con diagnóstico de fractura de cadera por fragilidad, definida como aquélla que fue consecuencia de un traumatismo de baja energía. Se excluyeron los pacientes con fracturas en terreno patológico asociadas a neoplasias así como los pacientes en quienes el clínico a cargo decidió establecer un manejo conservador de la fractura o el paciente falleció en el periodo de preparación para cirugía. Se dividió a los pacientes en dos grupos: con demora quirúrgica y sin demora quirúrgica. Se definió demora quirúrgica a los pacientes que fueron intervenidos en 48 horas o más desde su ingreso al servicio de urgencias. Se midieron las variables sociodemográficas de los pacientes, comorbilidades, funcionalidad, tipo de fractura, demora quirúrgica, dispositivo utilizado, estancia hospitalaria y principales resultados. Los datos de los pacientes se obtuvieron del expediente físico así como electrónico a través del sistema médico financiero (SIMEF). Se obtuvo autorización del comité local de 
ética e investigación con número de folio 138-2021. Se dividió a los pacientes en dos grupos: pacientes con cirugía temprana o en menos de 48 horas y pacientes con cirugía en más de 48 horas.

\section{Análisis estadístico}

Se reportan los resultados de variables categóricas en número y porcentaje. En el caso de variables continuas se reportan en medias \pm desviaciones estándar o mediana con rango intercuartil dependiendo del comportamiento en las pruebas de normalidad. Se compararon las variables continuas entre ambos grupos mediante t Student, y las variables categóricas mediante la prueba de $\chi^{2}$, $U$ de Mann Whitney 0 prueba exacta de Fisher, según corresponda.

El modelo actual de atención consta de una valoración por triaje en urgencias, dando aviso al servicio de traumatología y ortopedia. Una vez diagnosticada la fractura, se notifica al equipo interconsultante de ortogeriatría, quien realiza la valoración preoperatoria en las primeras 24 horas de estancia. En el caso de que el paciente ingresara en fin de semana, la valoración preoperatoria se realiza por el equipo de medicina interna. Se hacen los estudios preoperatorios y se optimizan condiciones para la programación quirúrgica. En este momento se busca la programación quirúrgica basada en disponibilidad de quirófano y material. Sólo se operaron pacientes en turno matutino y vespertino de lunes a viernes.

\section{Análisis de costos}

Los costos relacionados con la atención de $\mathrm{FC}$ se basó en contratos vigentes del instituto al momento de la realización del estudio obtenidos de la dirección normativa de administración y finanzas, subdirección de infraestructura. El material de osteosíntesis se obtuvo del servicio integral de osteosíntesis y endoprótesis ortopédica en las unidades médicas hospitalarias del ISSSTE contrato LPNE-CS-DA-SRMS-250/2017. Los costos asociados a la hospitalización se obtuvieron del tabulador de cuotas de recuperación por la atención médica de pacientes no derechohabientes del ISSSTE 2018. Los costos generales asociados al uso del inmueble, recursos humanos, consumibles e insumos por paciente se obtuvieron del documento Anexo A-I de la guía para elaborar un estudio técnico-médico ISSSTE 2015. El costo de los auxiliares de diagnóstico se obtuvo del contrato CAD/LEON/081/2019. Los costos derogados de los servicios de anestesia se obtuvieron de los servicios integrales de anestesia vigentes en el instituto.

Se realizó una estimación de costos relacionados con la intervención (auxiliares en el diagnóstico, estancia hospitalaria, material de osteosíntesis, recursos humanos e infraestructura hospitalaria) y se compararon entre los grupos con demora quirúrgica y sin demora. Como análisis de robustez se excluirán del análisis los costos comunes entre ambos grupos, dejando exclusivamente los costos sanitarios que varían entre grupos. Se usó un modelo de regresión así como modelos lineales generalizados ajustados a covariables de interés como sexo, edad, funcionalidad y riesgo anestésico.

\section{Resultados}

En el periodo comprendido entre el 01 de enero de 2017 y el 31 de diciembre de 2018 se ingresó un total de 88 fracturas de cadera por fragilidad. Se excluyeron cuatro pacientes por manejo conservador y dos pacientes fallecieron antes de la cirugía, quedando 82 para el análisis. Se muestran las características sociodemográficas en la Tabla 1. La edad media fue de 79.3 años con predominio de género femenino. La mayoría de los pacientes tenían marcha independiente y riesgo quirúrgico alto. Se encontraron diferencias estadísticamente significativas entre los grupos de estudio en la edad $(79.3 \pm 9.67 \mathrm{vs}$ $73 \pm 12 ; p=0.023)$, movilidad independiente $(91.7 \%$ vs $86.6 \% ; p=0.019)$ y riesgo quirúrgico alto $(50 \%$ vs $78.6 \% ; p=0.046)$. Sin embargo, dentro los pacientes con riesgo quirúrgico alto (48), sólo $12.7 \%$ de los retrasos para cirugía se atribuyeron a inestabilidad clínica $(p<0.001)$.

Con respecto al tipo de fractura y los resultados de la fase aguda (Tabla 2), no hubo diferencia entre el tipo de fracturas ni el dispositivo utilizado en cada una de ellas. Tampoco se encontró diferencia significativa en la técnica anestésica empleada. La media de demora quirúrgica en el grupo de cirugía tardía fue de 143 horas y en el grupo de cirugía temprana. Hubo diferencia estadísticamente significativa en la estancia hospitalaria (cuatro vs ocho días; $p=0.001$ ). Las principales causas de demora quirúrgica fueron falta de tiempo quirúrgico $(81.4 \%)$, falta de material (7.1\%), inestabilidad clínica (8.5\%) y anticoagulación (1.4\%). Los resultados asistenciales se muestran en la Tabla 2. Destaca un mayor porcentaje de úlceras por presión, neumonías y mortalidad, aunque no se encontró diferencia estadísticamente significativa. 
Tabla 1: Características basales de los pacientes con fractura de cadera por fragilidad.

\begin{tabular}{|c|c|c|c|c|}
\hline & $\begin{array}{l}\text { General } \\
(N=82)\end{array}$ & $\begin{array}{c}\text { Sin demora } \\
\text { quirúrgica }(N=12)\end{array}$ & $\begin{array}{l}\text { Con demora quirúrgica } \\
\qquad(\mathrm{N}=70)\end{array}$ & $p$ \\
\hline Edad (años) & $79.3 \pm 9.67^{\star}$ & $73 \pm 12^{*}$ & $80 \pm 9.2^{*}$ & $0.023^{\ddagger}$ \\
\hline Género femenino & $56(68.3)$ & $9(75)$ & $47(67.1)$ & $0.307 \S$ \\
\hline Funcionalidad & $90(60-95)$ & $88.3(85-100)$ & $85(57-93)$ & $0.06 \|$ \\
\hline Movilidad independiente ${ }^{* *}$ & $46(64)$ & $8(91.7)$ & $38(86.6)^{\ddagger \ddagger}$ & $0.019^{\S}$ \\
\hline Riesgo quirúrgico alto§§ & $61(74)$ & $6(50)$ & $55(78.6)$ & 0.046 ก \\
\hline Tipo de fractura & & & & $0.357 \S$ \\
\hline Subcapital & $25(30.5)$ & $5(41.6)$ & $20(28.6)$ & \\
\hline Transtrocantérea & $48(58.5)$ & $6(50)$ & $42(60)$ & \\
\hline Subtrocantérea & $9(11)$ & $1(8.3)$ & $8(11.4)$ & \\
\hline Tratamiento para osteoporosis prefractura & $9(11)$ & $1(8.3)$ & $8(11.4)$ & $0.608^{\S}$ \\
\hline Fracturas previas por fragilidad & $25(30.5)$ & $5(41.7)$ & $20(28.6)$ & $0.277^{\S}$ \\
\hline Diabetes mellitus & $4(33.3)$ & $4(33.3)$ & $29(41.4)$ & $0.423 \S$ \\
\hline Hipertensión & $8(66.7)$ & $8(66.7)$ & $42(60)$ & $0.460 \S$ \\
\hline Enfermedad renal crónica & $1(8.3)$ & $1(8.3)$ & $15(21.4)$ & $0.267 \S$ \\
\hline Historial de cáncer & $2(16.7)$ & $2(16.7)$ & $12(17.1)$ & $0.666^{\S}$ \\
\hline Enfermedades de tejido conectivo & $1(8.3)$ & $1(8.3)$ & $5(7.1)$ & $0.626^{\S}$ \\
\hline Historial de enfermedad vascular cerebral & $3(25.0)$ & $3(25.0)$ & $22(31.3)$ & $0.470 \S$ \\
\hline Deterioro cognitivo & $2(16.7)$ & $2(16.7)$ & $13(18.6)$ & $0.619 \S$ \\
\hline Fragilidad & $7(58.3)$ & $7(58.3)$ & $35(50)$ & 0.414 กศ \\
\hline Índice de comorbilidad de Charlson & $5(4-7)$ & $5 \pm 2^{*}$ & $5(4-7)^{\star * *}$ & $0.352 \|$ \\
\hline
\end{tabular}

Se reportan los resultados en $\mathrm{n}(\%)$, a menos que se especifique medias o medianas.

* Media \pm desviación estándar; ₹ t de Student; § prueba exacta de Fisher; ๆ índice de Barthel; |l prueba U de Mann Whitney; ** Categoría funcional para deambulación 3 a 5;

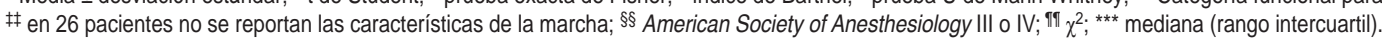

El costo atribuido a la atención a la fractura de cadera en nuestro hospital fue de $\$ 124,956.59$ pesos. Los costos desglosados en los pacientes con demora y sin demora se muestran en la Tabla 3. No se consideraron diferencias entre los auxiliares de diagnóstico ni en los dispositivos de osteosíntesis o prótesis implantados. Se encontró diferencia estadísticamente significativa en los costos totales ( $\$ 109,094.9$ vs $\$ 131,439 ; p=0.007)$, así como en los costos asociados exclusivamente a la hospitalización con una diferencia entre grupos de $\$ 25,539.10$ pesos mexicanos por evento. Al hacer ajustes multivariados para género-edad y riesgo anestésico se encontró una diferencia de $\$ 32,642$ y $\$ 28,418$, respectivamente con diferencia estadísticamente significativa. Al hacer el ajuste para funcionalidad la diferencia fue de $\$ 15,956$ sin significancia estadística (Figura 1).

\section{Discusión}

Hasta el momento del envío a publicación del presente trabajo, los autores no conocen ningún artículo o reporte sobre los costos asociados a la demora qui- rúrgica en México. El costo directo total de atención para fractura de cadera en nuestro hospital fue de $\$ 124,956.59$. Al comparar los grupos con demora contra pacientes con cirugía temprana, encontramos que existe una diferencia por cada caso de demora mayor de 48 horas de $\$ 25,359.10$, que si lo multiplicamos por el total de casos operados en este tiempo nos da un probable ahorro de $\$ 1,775,131$ en dos años. Este ahorro equivale a la atención de 225 adultos con hipertensión ${ }^{23}$ o 40 pacientes diabéticos por todo un año, ${ }^{24}$ además el costo por paciente de fractura de cadera es 3.5 veces mayor que el promedio del costo hospitalario en adultos mayores en un hospital de segundo nivel. ${ }^{25}$

Dentro de los resultados del estudio, observamos una diferencia estadísticamente significativa en la estancia hospitalaria con cuatro días de diferencia entre grupos, lo que le ahorraría al hospital un aproximado de 140 días/cama anualmente. Esto sin considerar los costos atribuidos a las complicaciones como úlceras por presión, neumonías o delirium, mismas que fueron mayores en el grupo de la demora quirúrgica. Un estudio en 57 pacientes postquirúrgicos reveló 
que el delirium incrementó el gasto de los servicios quirúrgicos en $\$ 11,762$ dólares estadounidenses. ${ }^{26}$ Por otra parte, se ha reportado que las infecciones hospitalarias incrementan hasta 11 días la estancia y hasta $\$ 2,000$ dólares estadounidenses. ${ }^{27}$ Otro estudio en España reveló que las úlceras por presión incrementan el costo asistencial desde $€ 2,400$ hasta $€ 9,000$ euros. ${ }^{28}$ Nuestro estudio reveló que estas complicaciones son las más frecuentes y consideramos que tienen relación con la demora quirúrgica, estancia hospitalaria y otros factores, con un impacto asistencial y económico relevante.

Otro punto a resaltar es que no hubo una diferencia significativa en la mayoría de las características prequirúrgicas entre los pacientes que se operaron de forma temprana y aquéllos con una demora quirúrgica mayor de 48 horas, exceptuando riesgo quirúrgico alto. Sin embargo, al analizar individualmente el motivo de la demora en pacientes con alto riesgo perioperatorio, la causa continúa siendo administrativa, lo que hace pensar que es un factor modificable. Este fenómeno es consistente con los hallazgos del proyecto del Registro Mexicano de Fractura de Ca- dera (ReMexFC) y de la Unidad de Ortogeriatría del Hospital General de San Luis Potosí, ambos trabajos reportan que la principal causa de demora es administrativa o de insumos y no atribuida a la condición clínica del paciente. ${ }^{12,19}$

Los hallazgos de ahorro en costos observados en nuestro estudio son consistentes con lo publicado en otros centros tanto en economías desarrolladas como emergentes. Israel descubrió que a pesar de gastar una mayor cantidad de recursos en la estabilización del paciente y en los procesos que permitan cirugía temprana, operar en las primeras 48 horas tiene un mayor impacto costo-beneficio. ${ }^{29}$ Otro estudio en el Reino Unido encontró que operar a los pacientes el día del ingreso o al día siguiente ahorra costos y tiempo de estancia independientemente del dispositivo protésico o de la osteosíntesis que se utilice. ${ }^{30}$ Por último, los beneficios económicos de tener una cirugía temprana se pueden extender a los costos en salud en el mediano y largo plazo. ${ }^{31}$

Con respecto a la diferencia de costos entre cirugía temprana y tardía, tras hacer ajustes con respecto

Tabla 2: Resultados asistenciales, tipo de cirugía e indicadores de calidad y complicaciones postquirúrgicas.

\begin{tabular}{|c|c|c|c|}
\hline & Sin demora quirúrgica $(\mathrm{N}=12)$ & Con demora quirúrgica $(\mathrm{N}=70)$ & $\mathrm{p}$ \\
\hline Tipo de cirugía & & & $0.983^{*}$ \\
\hline Prótesis parcial & $5(41.7)$ & $26(37.1)$ & \\
\hline Prótesis total & - & $4(5.7)$ & \\
\hline DHS & $6(50.0)$ & $25(35.7)$ & \\
\hline Otro & $1(8.3)$ & $11(15.9)$ & \\
\hline Tipo de anestesia & & & $0.854^{*}$ \\
\hline Regional & $12(100)$ & $69(98.6)$ & \\
\hline General & - & $1(1.4)$ & \\
\hline Demora quirúrgica en horas & $42 \pm 4.6$ & $143(91-168)$ & $0.140^{\ddagger}$ \\
\hline Sedestación postquirúrgica & $7(58.3)$ & $19(27.1)$ & $0.050^{\S}$ \\
\hline Destino al alta & & & $1.000^{*}$ \\
\hline Domicilio & $12(100)$ & $65(92.9)$ & \\
\hline Estancia hospitalaria & $4(4-6)^{\mathbb{4}}$ & $8(6-10)^{\pi}$ & $0.001^{\ddagger}$ \\
\hline Tratamiento para osteoporosis al alta & $5(41.7)$ & $16(22.9)$ & $0.153^{\S}$ \\
\hline Complicación mayor & $9(75.0)$ & $47(67.1)$ & $0.430^{*}$ \\
\hline Neumonía & - & $9(12.9)$ & $0.222^{*}$ \\
\hline Úlcera por presión & $2(16.7)$ & $22(31.4)$ & $0.250^{*}$ \\
\hline Infección urinaria & $2(16.7)$ & $11(15.7)$ & $0.600^{*}$ \\
\hline Anemia postoperatoria & $6(50.0)$ & $34(48.6)$ & $0.580^{\S}$ \\
\hline Delirium & $6(50.0)$ & $31(44.3)$ & $0.470^{\S}$ \\
\hline Mortalidad hospitalaria & - & $3(4.3)$ & $0.610^{*}$ \\
\hline
\end{tabular}

Se reportan los resultados en $\mathrm{n}(\%)$, a menos que se especifique medias o medianas.

${ }^{\star}$ Prueba exacta de Fisher; ${ }^{\ddagger}$ prueba U de Mann Whitney; ${ }^{\S} \chi^{2} ;{ }^{\Uparrow}$ mediana (rango intercuartil); — ningún paciente recibió rehabilitación postoperatoria.

DHS = Dynamic hip screw. 
Tabla 3: Costos de la atención de fractura de cadera por fragilidad.

\begin{tabular}{llcl}
\hline & Con demora $(\mathrm{N}=70)$ & Sin demora $(\mathrm{N}=12)$ & $\mathrm{p}^{*}$ \\
\hline Costo total & $\$ 128,694.3 \pm 37,588.33$ & $\$ 103,155.2 \pm 22,724.86$ & 0.025 \\
Material & $\$ 46,831.98 \pm 9,862.48$ & $\$ 47,113.18 \pm 6,735.36$ & 0.924 \\
Hospitalización & $\$ 54,293.14 \pm 25,879.55$ & $\$ 33,150 \pm 17,786.97$ & 0.008 \\
\hline
\end{tabular}

Se reportan medias \pm desviación estándar. Costos reportados en pesos mexicanos al año 2018.

* U de Mann-Whitney.

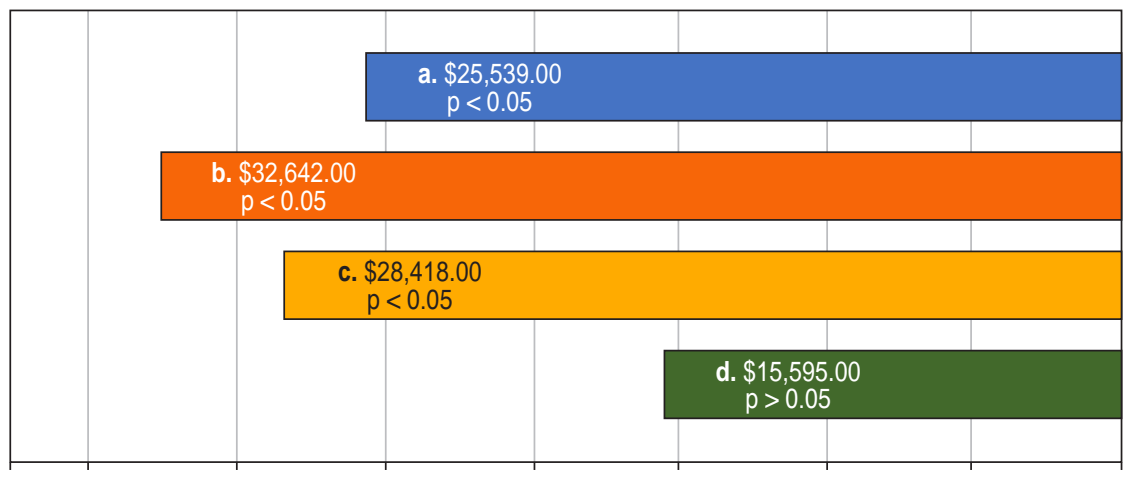

$\$ 35,000.00 \$ 30,000.00 \$ 25,000.00 \$ 20,000.00 \$ 15,000.00 \$ 10,000.00 \$ 5,000.00$

Pesos mexicanos
Figura 1:

Costos totales de la fractura de cadera y modelos de ajuste. Cirugía en menos de 48 horas contra más de 48 horas. Modelos ajustados a variables específicas. a) Diferencia entre el grupo con demora y sin demora. b) Ajuste con edad y género. c) ajuste con riesgo quirúrgico. d) Ajuste con funcionalidad (Barthel). a edad, género y riesgo quirúrgico, la tendencia hacia el ahorro se mantiene, lo que nos hace pensar que el posible ahorro sería consistente independientemente del perfil de los pacientes atendidos con fractura de cadera. Por otra parte, las principales causas de demora fueron la falta de tiempo quirúrgico y la falta de material. Este problema se puede atribuir, en opinión de los autores, a dos fenómenos que se potencian entre sí. El primero es a que al ser un hospital público, frecuentemente se encuentra cercano a su límite de capacidad instalada y con saturación de los servicios hospitalarios. El segundo es la falsa creencia de que la fractura de cadera no debe ser tratada como una urgencia quirúrgica.

La principal debilidad del estudio es que los costos individuales de los pacientes no se tienen sistematizados en un sistema electrónico que facilite el cálculo con menor margen de error; sin embargo, la estimación de costos está basada en la mayor cantidad de información disponible en el instituto. Consideramos que la principal fortaleza de este trabajo es ser el primer estudio mexicano que analiza la demora quirúrgica como factor modificable para ahorro de costos e impacto en resultados asistenciales.

\section{Conclusiones}

La demora quirúrgica en nuestro hospital tiene repercusiones negativas en los resultados asistenciales, además de incrementar de manera innecesaria los costos en salud. Estos costos asociados a la demora quirúrgica no se pueden atribuir a la edad, género o riesgo quirúrgico, por lo que se convierten en un factor modificable en el proceso de atención. Aunado a esto, las principales causas de demora no son inherentes al estado clínico del paciente, por lo que también pueden considerarse un factor modificable en la atención. Se deben generar políticas internas y mejora en los procesos, considerando la fractura de cadera una urgencia meritoria de cirugía temprana, llevando a una mejora potencial en resultados así como un ahorro significativo en costos para las instituciones públicas en México.

\section{Referencias}

1. Organización Mundial de la Salud. Informe mundial sobre envejecimiento y salud. Luxemburgo: OMS; 2015. [Último acceso 02 junio 2021] Disponible en: https://www.who.int/ ageing/publications/world-report-2015/es/ 
2. Instituto Nacional de Estadística Geografía e Informática (INEGI). Censo Instituto Nacional de Estadística Geografía e Informática. Porcentaje de hombres de 60 y más años. 2019. [Último acceso 02 junio 2021] Disponible en: https://www.inegi. org.mx/app/buscador/default.html?q=poblacion+mayo+de+60 tan?os\#tabMCcollap se-Indicadores

3. International Osteoporosis Foundation. The Latin American Regional Audit. Epidemiología, costos e impacto de la osteoporosis en 2012. 2012. [Ultimo acceso 02 junio 2021] Disponible en: https://www.osteoporosis.foundation/sites/iofbonehealth/ files/201906/2012_Latin_America_Audit_Espanol.pdf

4. Intervenciones de enfermería para la atención de adultos mayores con fractura de cadera. Guía de Evidencias y Recomendaciones: Guía de Práctica Clínica. México: Instituto Mexicano del Seguro Social; 2018. [Último acceso junio 2021] Disponible en: https://www.imss.gob.mx/sites/all/statics/ guiasclinicas/603GER.pdf

5. Guzon-Illescas O, Perez Fernandez E, Crespí Villarias N, Quirós Donate FJ, Peña M, Alonso-Blas C, et al. Mortality after osteoporotic hip fracture: incidence, trends, and associated factors. J Orthop Surg Res. 2019; 14 (1): 203.

6. Veronese N, Maggi S. Epidemiology and social costs of hip fracture. Injury. 2018; 49 (8): 1458-1460.

7. Edelmuth SVCL, Sorio GN, Sprovieri FAA, Gali JC, Peron SF. Comorbidities, clinical intercurrences, and factors associated with mortality in elderly patients admitted for a hip fracture. Rev Bras Ortop. 2018; 53 (5): 543-551.

8. Cooper C, Cole ZA, Holroyd CR, Earl SC, Harvey NC, Dennison EM, et al. Secular trends in the incidence of hip and other osteoporotic fractures. Osteoporos Int. 2011; 22 (5): $1277-1288$.

9. International Osteoporosis Foundation. Epidemiology of fragility fractures. [Ultimo acceso 02 junio 2021] Available in: https:// www.osteoporosis.foundation/facts-statistics/epidemiology-ofosteoporosis-and-fragility-fractures

10. Klestil T, Roder C, Stotter C, Winkler B, Nehrer S, Lutz M, et al. Impact of timing of surgery in elderly hip fracture patients: a systematic review and meta-analysis. Sci Rep. 2018; 8 (1): 13933.

11. Anthony CA, Duchman KR, Bedard NA, Gholson JJ, Gao Y, Pugely AJ, et al. Hip fractures: appropriate timing to operative intervention. J Arthroplasty. 2017; 32 (11): 3314-3318.

12. Viveros-García JC, Robles-Almaguer E, Arechiga Muñoz E, López Cervantes RE, Torres-Naranjo JF, Baldenebro-Lugo LS. Mexican hip fracture audit (ReMexFC): pilot phase report. J Lat Am Geriatric Med. 2020; 6 (1): 1-9.

13. Chang W, Lv H, Feng C, Yuwen P, Wei N, Chen W, et al. Preventable risk factors of mortality after hip fracture surgery: systematic review and meta-analysis. Int J Surg. 2018; 52: 320-328.

14. Villalon-Coca J, Romero-García E, Leira S, Cerda-Palau A, Rollan-García P. La estancia prequirúrgica en la fractura de cadera: la perspectiva del gasto. Gest y Eval Costos Sanit. 2016; 17 (2): 121-131.

15. Farrow L, Hall A, Wood AD, Smith R, James K, Holt G, et al. Quality of care in hip fracture patients: the relationship between adherence to national standards and improved outcomes. J Bone Joint Surg Am. 2018; 100 (9): 751-757.

16. Freeman C, Todd C, Camilleri-Ferrante C, Laxton C, Murrell P, Palmer CR, et al. Quality improvement for patients with hip fracture: experience from a multi-site audit. Qual Saf Health Care. 2002; 11 (3): 239-245.
17. National Institute for Health and Care Excellence (NICE). Hip fracture management guidelines. 2011 updated 2017. [Ultimo acceso 04 junio 2021] Disponible en: https://www.nice.org. uk/guidance/cg124/resources/hip-fracture-managementpdf-35109449902789

18. Candelario Martínez LJ, López Esqueda FJ, González González GR, González Rubio MV, García Aguirre N, Shiguetomi Medina $\mathrm{JM}$. Associated factors to surgical delay hip fracture in a Mexican hospital. J Lat Am Geriatric Med. 2019; 5 (2): 33-38.

19. Quevedo-Tejero EC, Zavala-Gonzalez MA, Hernández-Gamas AC, Hernández-Ortega HM. Hip fracture in older adults: prevalence and costs in two hospitals. Tabasco, Mexico, 2009. Rev Peru Med Exp Salud Publica. 2011; 28 (3): 440-445.

20. Carlos F, Clark P, Maciel H, Tamayo JA. Direct costs of osteoporosis and hip fracture: an analysis for the Mexican Social Insurance Health Care System. Salud Publica Mex. 2009; 51 Suppl 1: S108-S113.

21. Clark P, Carlos F, Barrera C, Guzman J, Maetzel A, Lavielle P, et al. Direct costs of osteoporosis and hip fracture: an analysis for the Mexican healthcare system. Osteoporos Int. 2008; 19 (3): 269-276.

22. Clark P, Carlos F, Vázquez Martínez JL. Epidemiology, costs and burden of osteoporosis in Mexico. Arch Osteoporos. 2010; 5 (1-2): 9-17.

23. Villareal-Rios E, Mathew-Quiroz A, Garza-Elizondo ME, Nuñez-Rocha G, Salinas-Martinez AM, Gallegos-Handal G. Costo de la atención a la hipertensión arterial y su impacto en el presupuesto destinado en saludo en México. Salud Pública Mex. 2002; 44: 7-13.

24. Macías-Sánchez A, Villareal-Paez HJ. Sostenibilidad del gasto público: Cobertura y financiamiento de enfermedades crónicas en México. Ensayos Revista de Economía. 2018; 37 (1): 99-134.

25. Granados-García V, Sánchez-García S, Ramírez-Aldana R, Zúñiga-Trejo C, Espinel-Bermúdez CE. Costos por hospitalización de adultos mayores en un hospital regional del IMSS. Rev Med Inst Mex Seguro Soc. 2018; 56 (1): S64-S70.

26. Franco K, Litaker D, Locala J, Bronson D. The cost of delirium in the surgical patient. Psychosomatics. 2001; 42 (1): 68-73.

27. Arreguin-Nava R, González-González R, De la Torre-Rosas A. Infecciones adquiridas en los hospitales. ¿Cuánto cuestan y como se calcula? Rev Digit Univ. 2012; 13 (9): 1-10.

28. Soldevilla Agreda JJ, Torra I Bou JE, Posnett J, Verdu Soriano J, San Miguel L, Mayan Santos M. The burden of pressure ulcers in Spain. Wounds. 2007; 19 (7): 201-206.

29. Shabat S, Heller E, Mann G, Gepstein R, Fredman B, Nyska M. Economic consequences of operative delay for hip fractures in a non-profit institution. Orthopedics. 2003; 26 (12): 1197-1199; discussion 1199.

30. Castelli A, Daidone S, Jacobs R, Kasteridis P, Street AD. The determinants of costs and length of stay for hip fracture patients. PLoS ONE. 2015; 10 (7): e0133545.

31. Pincus D, Wasserstein D, Ravi B, Huang A, Paterson M, Jenkinson R, et al. Medical costs of delayed hip fracture surgery. J Bone Joint Surg Am. 2018; 100: 1387-1396.

\section{Conflicto de intereses}

Los autores declaran no tener ningún conflicto de intereses. 